\title{
Anti-angiogenesis gene therapy for hepatocellular carcinoma via systemic injection of mesenchymal stem cells engineered to secrete soluble Flt-1
}

\author{
GUANGLIN LI $^{1}$, FEI MIAO ${ }^{2}$, JINHAI ZHU ${ }^{1}$ and YANLING CHEN ${ }^{1}$ \\ ${ }^{1}$ Department of Hepatobiliary Surgery and Fujian Institute of Hepatobiliary Surgery, \\ Fujian Medical University Union Hospital; ${ }^{2}$ Department of Obstetrics and Gynecology, \\ The First Hospital of Fuzhou, Fuzhou, Fujian 350001, P.R. China
}

Received October 27, 2016; Accepted June 19, 2017

DOI: $10.3892 / \mathrm{mmr} .2017 .7310$

\begin{abstract}
Anti-angiogenesis gene therapy has attracted interest as a potential treatment for hepatocellular carcinoma (HCC). Studies have indicated that soluble fms-like tyrosine kinase-1 (sFlt-1) may suppress angiogenesis by sequestering free vascular endothelial growth factor (VEGF) or by forming inactive heterodimers with VEGF receptor-2. Mesenchymal stem cells (MSCs) have been widely used as prospective delivery vehicles for therapeutic agents, owing to their ability to migrate towards tumor sites. In the present study, a subcutaneous HCC mouse model was used to assess the anti-angiogenesis effects of lentivirus-transfected MSCs engineered to secrete sFlt-1 (LV-sFlt-1-MSCs). LV-sFlt-1-MSCs effectively secreted sFlt-1, which inhibited tube formation in vitro. MSCs labeled with green fluorescence protein primarily migrated to tumor sites in vivo. An immunohistochemical assay indicated that microvessel density was reduced in mice treated with LV-sFlt-1-MSCs, compared with the control group treated with PBS. Additionally, LV-sFlt-1-MSCs inhibited tumor growth and prolonged survival in an HCC mouse model via systemic injection. Overall, the present study was designed to investigate the potential of LV-sFlt-1-MSCs for anti-angiogenesis gene therapy in HCC.
\end{abstract}

\section{Introduction}

Hepatocellular carcinoma (HCC) is a common lethal malignancy that is highly vascularized and is the third most

Correspondence to: Professor Jinhai Zhu, Department of Hepatobiliary Surgery and Fujian Institute of Hepatobiliary Surgery, Fujian Medical University Union Hospital, 29 Xinquan Road, Fuzhou, Fujian 350001, P.R. China

E-mail: drzhujinhai@163.com

Key words: mesenchymal stem cells, vascular endothelial growth factor, anti-angiogenesis, soluble fms-like tyrosine kinase 1, hepatocellular carcinoma common cause of cancer-associated mortality worldwide (1). Angiogenesis is important for tumor progression and metastasis; it is a complex process regulated by a variety of factors, among which vascular endothelial growth factor (VEGF) appears to serve a dominant role (2). Previous studies have indicated that VEGF is overexpressed in HCC and is closely associated with tumor angiogenesis $(3,4)$. Therefore, targeting the VEGF/VEGF receptor (VEGFR) signaling pathway has attracted attention in anti-angiogenesis therapy research.

The soluble form of VEGFR-1 (also known as soluble fms-like tyrosine kinase-1, sFlt-1), which is an alternatively spliced variant, has been hypothesized to be a potent antagonist of VEGF. Full-length VEGFR-1 is a glycoprotein with seven extracellular immunoglobulin (Ig)-like domains, a transmembrane domain and an intracellular tyrosine kinase binding domain $(5,6)$. The spliced sFlt-1 comprises only the first six extracellular Ig-like domains (7). Although VEGFR-1 has an increased affinity for VEGF compared with VEGFR-2, the tyrosine kinase activity of VEGFR-2 is more effective by a factor of ten (8). VEGFR-2 is considered to be the primary receptor involved in angiogenesis. Despite having the same affinity as VEGFR-1 for VEGF, sFlt-1 does not initiate signal transduction, owing to the absence of the transmembrane domain and the intracellular tyrosine kinase domain. sFlt-1 exerts anti-angiogenesis effects in two ways: i) Sequestering free ligands; and ii) forming inactive heterodimers with VEGFR-2 (9). A variety of studies have demonstrated that the administration of sFlt-1 may suppress tumor growth and metastasis through anti-angiogenesis mechanisms (10-13). However, gene therapy remains limited, as therapeutic molecules that are encoded by the target gene may not effectively localize to tumor sites following systemic injection.

Mesenchymal stem cells (MSCs) are immune privileged pluripotent cells (14) that are easy to isolate and culture in vitro. In addition to their multilineage differentiation potential, MSCs have been observed to migrate to the site of wounds and tumors (15), including glioma (16), hepatoma and lung tumors (17). These features make MSCs an ideal delivery vehicle for gene therapy, and MSCs engineered to carry therapeutic genes have been demonstrated to serve an antitumor role in certain tumor models (18-22). 
The present study evaluated the anti-angiogenic effects of lentiviral transfected MSCs engineered to secrete sFlt-1 (LV-sFlt-1-MSCs) via co-culturing of these cells with human umbilical vein endothelial cells (HUVECs) in a three-dimensional co-culture system. To investigate the antitumor effects of sFlt-1 and the tropism of MSCs for the tumor site, LV-sFlt-1-MSCs were produced by lentiviral transduction and were implanted into mice as part of a pre-established HCC subcutaneous mouse model. The results of the present study suggested that MSCs may serve as delivery vehicles for gene therapy in HCC.

\section{Materials and methods}

Cell culture. The HCC cell line SMMC-7721 was purchased from Shanghai Institute of Biochemistry and Cell Biology, Chinese Academy of Science (Shanghai, China). BALB/C mouse bone marrow-derived MSCs were obtained from Cyagen Biosciences (Santa Clara, CA, USA). SMMC-7721 cells were maintained in Dulbecco's modified Eagle's medium (DMEM; Gibco; Thermo Fisher Scientific, Inc., Waltham, MA, USA). MSCs were incubated in DMEM/Ham's F12 nutrient mixture (HyClone; GE Healthcare Life Sciences, Logan, UT, USA). All cell cultures were supplemented with $10 \%$ fetal bovine serum (FBS; Gibco; Thermo Fisher Scientific, Inc.), $100 \mu \mathrm{g} / \mathrm{ml}$ streptomycin and $100 \mathrm{U} / \mathrm{ml}$ penicillin (Gibco; Thermo Fisher Scientific, Inc.), and incubated at $37^{\circ} \mathrm{C}$ in a $5 \%$ $\mathrm{CO}_{2}$ atmosphere. HUVECs (ScienCell Research Laboratories, Inc., Carlsbad, CA, USA) were incubated in endothelial growth medium (PromoCell GmbH, Heidelberg, Germany) supplemented with 5\% FBS. MSCs and HUVEC were used during the third or fourth passage.

Transfection of MSCs. The lentiviral vectors expressing sFlt-1 (LV-sFlt-1, 9x10 $10^{8} \mathrm{IU} / \mathrm{ml}$ ) and non-targeting control lentiviral

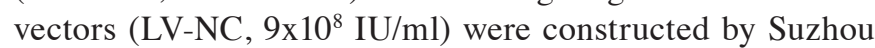
GenePharma Co., Ltd. (Suzhou, China). MSCs $\left(5 \times 10^{5}\right.$ cells $\left./ \mathrm{ml}\right)$ were transfected at $37^{\circ} \mathrm{C}$ for $72 \mathrm{~h}$ using Lipofectamine 2000 (Thermo Fisher Scientific, Inc.) according to the manufacturer's guidelines. The suitable multiplicity of infection was determined to be 50 in the present study. Stably infected MSCs were obtained by treating the cells with puromycin $(2 \mu \mathrm{g} / \mathrm{ml})$.

Semi-quantitative polymerase chain reaction (PCR). LV-sFlt-1-MSCs, LV-NC-MSCs and MSCs were cultured as aforementioned. Following $72 \mathrm{~h}$, cells $\left(1 \times 10^{6}\right.$ cells $\left./ \mathrm{ml}\right)$ were harvested by centrifugation at $1,000 \mathrm{x} \mathrm{g}$ at room temperature for $10 \mathrm{~min}$ and the supernatant was stored for ELISA analysis. Total RNA was isolated from cells using TRIzol (Invitrogen; Thermo Fisher Scientific, Inc.), following the manufacturer's instructions. Analysis was performed using PrimeSTAR HS DNA polymerase (Takara Biotechnology Co., Ltd., Dalian, China). Thermocycling conditions were as follows: Initial denaturation at $95^{\circ} \mathrm{C}$ for $5 \mathrm{~min}$, followed by 33 cycles of denaturation at $95^{\circ} \mathrm{C}$ for $30 \mathrm{sec}$, annealing at $55^{\circ} \mathrm{C}$ for $30 \mathrm{sec}$, extension at $72^{\circ} \mathrm{C}$ for $30 \mathrm{sec}$, with a final extension step at $72^{\circ} \mathrm{C}$ for $10 \mathrm{~min}$. The primers for sFlt-1 were as follows: Forward, 5'-GATGGGTTACCTGCGACTG-3' and reverse, 5'-CCG GGTCTGGAAACGATG-3'. The primers for GAPDH were as follows: Forward, 5'-AGGGCTGCTTTTAACTCTGGT-3' and reverse, 5'-TCTCGCTCCTGGAAGATGGTG-3'. PCR products were dissolved on a $12 \%$ agarose gel and visualized following staining with ethidium bromide $(0.5 \mu \mathrm{g} / \mathrm{ml}$; Thermo Fisher Scientific, Inc.). Densitometric analysis was performed using ImageJ software version 1.45 (National Institutes of Health, Bethesda, MD, USA).

Western blot analysis. Cells $\left(1 \times 10^{6}\right.$ cells $\left./ \mathrm{ml}\right)$ were lysed in radioimmunoprecipitation assay buffer (Beyotime Institute of Biotechnology, Haimen, China) with phenylmethylsulfonyl fluoride (a protease inhibitor). Protein concentration was determined using a bicinchoninic acid protein assay. A total of $25 \mu \mathrm{g}$ total protein was separated by $10 \%$ SDS-PAGE and transferred onto polyvinylidene fluoride membranes. The membranes were blocked for $2.5 \mathrm{~h}$ with $5 \%$ non-fat milk at room temperature and incubated with monoclonal rabbit anti-human Flt-1 antibody (1:1,000, cat. no. ab32152; Abcam, Cambridge, UK) and monoclonal mouse anti-human $\beta$-actin antibody $(1: 1,000$, cat. no. sc-130065; Santa Cruz Biotechnology, Inc., Dallas, TX, USA) at $4^{\circ} \mathrm{C}$ overnight. Subsequently, the membranes were incubated with the secondary antibody conjugated with horseradish peroxidase $(1: 1,000$, cat. no. sc-516102; Santa Cruz Biotechnology, Inc.) for $1 \mathrm{~h}$ at room temperature. Immunoreactivity was detected using an enhanced chemiluminescence kit (Beyotime Institute of Biotechnology), in accordance with the manufacturer's instructions. $\beta$-actin served as an internal control. Blots were semi-quantified by densitometry using ImageJ software version 1.45.

Tube formation assay. To evaluate the role of sFlt-1 in the tube formation of HUVECs, LV-sFlt-1-MSCs, LV-NC-MSCs and untransfected MSCs were co-cultured with HUVECs following the method previously described by Zeng et al (23). A 15 -well $\mu$-slide (ibidi GmbH, Munich, Germany) was coated with Matrigel (10 $\mu \mathrm{l} /$ well; BD Biosciences, Franklin Lakes, NJ, USA) and incubated for $30 \mathrm{~min}$ at $37^{\circ} \mathrm{C}$. Subsequently, HUVECs $\left(7.5 \times 10^{3}\right.$ cells/well $)$ were stained with DiI $(10 \mu \mathrm{M}$, a cell membrane dye emitting red fluorescence; Beyotime Institute of Biotechnology) at $37^{\circ} \mathrm{C}$ for $30 \mathrm{~min}$. HUVECs and LV-sFlt-1-MSCs $\left(7.5 \times 10^{3}\right.$ cells/well) were plated in the same well of the $\mu$-slide at $37^{\circ} \mathrm{C}$. Images were digitally captured at 1 , $3,5,12$ and $24 \mathrm{~h}$ under a fluorescence microscope (x100 magnification) and analysis of tube formation was performed using Image J software version 1.45.

Distribution of MSCs in vivo. SMMC-7721 cells $\left(7 \times 10^{6}\right)$ were stained with $\mathrm{DiI}(10 \mu \mathrm{M})$ at $37^{\circ} \mathrm{C}$ for $30 \mathrm{~min}$ prior to subcutaneously injecting the cells into the rear of nude mice. Tumors were allowed to grow for 2 weeks; subsequently, $5 \times 10^{5}$ LV-NC-MSCs were intravenously injected into tumor-bearing mice via the tail vein. Following 7 days additional growth, the subcutaneous tumor tissues, livers, lungs, hearts, kidneys and brains were removed and cut into fresh-frozen $\left(-15^{\circ} \mathrm{C}\right)$ sections $(5 \mu \mathrm{m})$. To visualize the distribution of MSCs in vivo, the sections were observed under a fluorescence microscope (x200 magnification).

Animal experiments. Male athymic BALB/C nude mice (age, 4-6 weeks; weight, 18-22 g) were purchased from Shanghai SLAC Laboratory Animal Co., Ltd. (Shanghai, China) and 
maintained under specific pathogen free conditions at a temperature of $22-25^{\circ} \mathrm{C}$, under a 12-h light/dark cycle with free access to food and water. All experiments were performed in accordance with the National Institutes of Health Guide for the Care and Use of Laboratory Animals, and were approved by the Ethics Committee of the Medical Faculty of the Fujian Medical University (Fuzhou, China).

SMMC-7721 cells (7x10 $10^{6}$ cells in $0.1 \mathrm{ml}$ PBS) were subcutaneously implanted into the rear of nude mice. When the diameter of the tumor nodule reached $0.5 \mathrm{~cm}$, tumor-bearing mice were randomized into 4 groups ( $n=10 /$ group): i) PBS (0.1 ml PBS once a week for 3 weeks); ii) LV-NC-MSCs; iii) LV-sFlt-1; and iv) LV-sFlt-1-MSCs. LV-NC-MSCs or LV-sFlt-1-MSCs $\left(6 \times 10^{5}\right.$ cells in $\left.0.1 \mathrm{ml} \mathrm{PBS}\right)$ were intravenously injected via the tail vein once per week for 3 weeks. LV-sFlt-1 ( $3 \times 10^{7} \mathrm{TU}$ in $0.1 \mathrm{ml}$ PBS) was injected once per week for 3 weeks. Tumor size was measured using a caliper every 5 days. The length (L) and width (W) of tumors were measured, and tumor volume (V) was calculated using the following formula: $\mathrm{V}=\mathrm{L} \times \mathrm{W}^{2} / 2$. A total of 3 weeks later, 5 mice in each group were sacrificed. Blood samples were harvested from mouse hearts $(1.5 \mathrm{ml})$ and plasma was obtained by centrifugation at room temperature at $1,000 \mathrm{x} \mathrm{g}$ for $15 \mathrm{~min}$. Tumor tissues were removed and minced in PBS. Plasma samples and tumor tissues were stored at $-20^{\circ} \mathrm{C}$. The remaining mice were monitored for survival analysis.

ELISA analysis. Cell culture supernatants $(0.1 \mathrm{ml})$, plasma samples $(0.1 \mathrm{ml})$ and fresh tumor tissues $(0.1 \mathrm{ml})$ were harvested, and the concentration of secreted sFlt-1 was detected by ELISA for human VEGFR1/Flt-1 (cat. no. DVR100B; R\&D Systems Inc., Minneapolis, MN, USA), according to the manufacturer's instructions.

Immunohistochemistry. Tumor tissues were fixed in $4 \%$ paraformaldehyde at room temperature for $24 \mathrm{~h}$ and embedded in paraffin. Sections $(4 \mu \mathrm{m})$ were cut, dewaxed and dehydrated. Subsequently, sections were stained with $0.2 \%$ hematoxylin for $5 \mathrm{~min}$ and $1 \%$ eosin for $2 \mathrm{~min}$, and observed under an optical microscope (x400 magnification). Immunohistochemical staining was also performed. Tissue sections were blocked in 5\% goat serum (Gibco; Thermo Fisher Scientific, Inc.) diluted in PBS for $20 \mathrm{~min}$ at room temperature. The primary antibodies included: Monoclonal rabbit anti-murine cluster of differentiation (CD)34 (1:50, cat. no. sc-9095; Santa Cruz Biotechnology, Inc.); monoclonal rabbit anti-human proliferation marker protein Ki-67 (Ki-67, 1:50, cat. no. sc-15402; Santa Cruz Biotechnology, Inc.); and polyclonal rabbit anti-human VEGF (1:250, cat. no. ab46154; Abcam). Sections were incubated with the primary antibodies at $4^{\circ} \mathrm{C}$ for $24 \mathrm{~h}$. After washing, the sections were incubated with a MaxVision ${ }^{\mathrm{TM}}$ horseradish peroxidase-labelled polymer anti-rabbit kit (cat. no. KIT-5005; Fuzhou Maixin Biotech Co., Ltd., Fuzhou, China) at room temperature for $15 \mathrm{~min}$. Finally, they were stained with 3,3'-diaminobenzidince (Fuzhou Maixin Biotech Co., Ltd.) at room temperature for $5 \mathrm{~min}$. Stained sections were observed and scored independently by two pathologists. The proliferation index was determined as the number of [Ki-67-positive (brown) cells/the number of total cells] x 100, in five random fields under an optical microscope (x400 magnification).
Microvessel density (MVD) analysis. MVD was assayed by immunohistochemical staining with a CD34 antibody under an optical microscope and was evaluated as previously reported by Weidner et al (24). The sections were visualized under low magnification (x100) to identify the highest neovascularization areas (hotspots). Subsequently, these areas were observed at higher magnification (x200) and the number of microvessels was counted in 5-random fields. The average number of microvessels in five fields was regarded as the MVD level for the sample.

Statistical analysis. The data are presented as the mean \pm standard error of the mean from at least 3 independent experiments. Survival data were analyzed using the Kaplan-Meier method, and statistical significance was determined using the log-rank test. Statistical significance was evaluated using one-way analysis of variance followed by a post hoc Dunnett's test for multiple comparisons. $\mathrm{P}<0.05$ was considered to indicate a statistically significant difference.

\section{Results}

Construction of LV-sFlt-1-MSCs and expression of sFlt-1 in vitro. MSCs were infected with LV-sFlt-1 or LV-NC, and stably infected MSCs were obtained by treating the cells with puromycin (Fig. 1A). To determine the expression levels of sFlt-1, PCR and western blotting were performed to analyze lysates from LV-sFlt-1-MSCs, LV-NC-MSCs and untransfected MSCs. LV-sFlt-1-MSCs significantly overexpressed sFlt-1 mRNA and protein compared with MSCs and LV-NC-MSCs (Fig. 1B-E). These stably infected MSCs were used for subsequent experiments.

sFlt-1 levels in cell culture supernatants, plasma samples and fresh tumor tissues. To determine whether sFlt-1 may be secreted by lentivirus-infected MSCs, an ELISA for human VEGFR1/Flt-1 was performed. The secreted sFlt-1 level in the culture supernatants of LV-sFlt-1-MSCs was $5793.0 \pm 73.9 \mathrm{pg} / \mathrm{ml}$, whereas only minimal sFlt-1 was detected in the culture supernatants of LV-NC-MSCs and untreated MSCs (Fig. 1F).

Human sFlt-1 levels in plasma samples and tumor tissues were undetectable in control mice treated with PBS or LV-NC-MSCs (data not shown). The plasma concentration of human sFlt-1 in the mice treated with LV-sFlt-1 was significantly higher compared with the concentration in mice treated with LV-sFlt-1-MSCs $(301.7 \pm 7.7 \mathrm{pg} / \mathrm{ml}$ vs. $19.2 \pm 2.2 \mathrm{pg} / \mathrm{ml}$; Fig. 1G). However, the intratumoral level of human sFlt-1 in the mice treated with LV-sFlt-1-MSCs was increased compared with the group treated with LV-sFlt-1 $(860.7 \pm 63.94 \mathrm{pg} / \mathrm{mg}$ vs. $14.1 \pm 8.79 \mathrm{pg} / \mathrm{mg}$; Fig. $1 \mathrm{H})$.

LV-sFlt-1-MSCs inhibit tube formation in vitro. To determine the bioactivity of sFlt-1 in vitro, HUVECs stained with DiI were co-cultured with LV-sFlt-1-MSCs, LV-NC-MSCs or MSCs in a three-dimensional co-culture system. The largest number of tubes was observed $5 \mathrm{~h}$ subsequent to co-culturing (Fig. 2A and B), and the tube-like structures disappeared at $24 \mathrm{~h}$ (data not shown). Tube formation was significantly decreased in the LV-sFlt-1-MSCs group compared with the LV-NC-MSC or MSC group. 
A

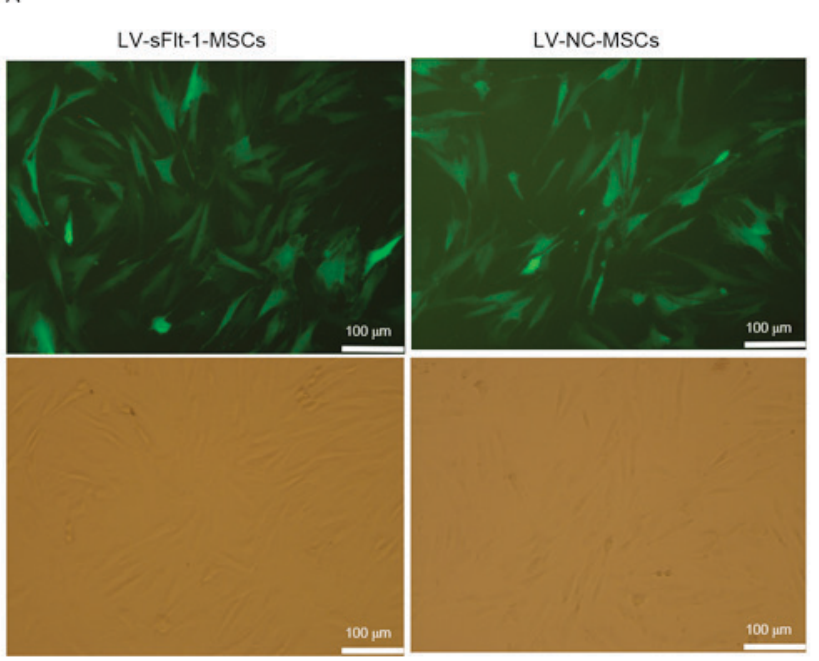

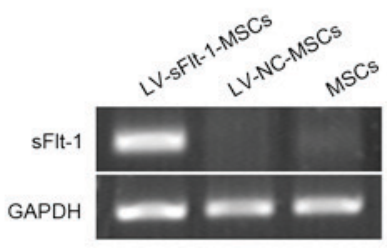

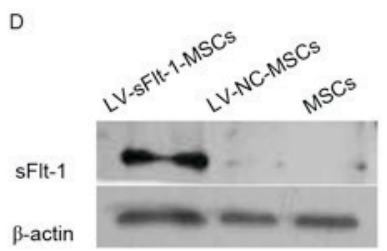

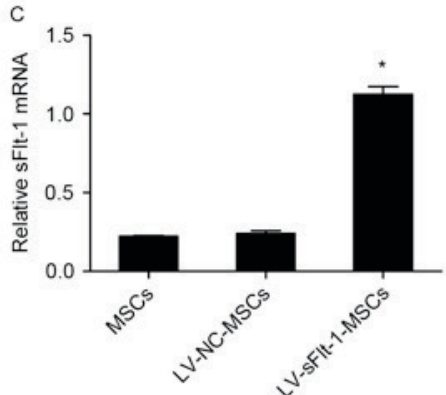

$\mathrm{E}$

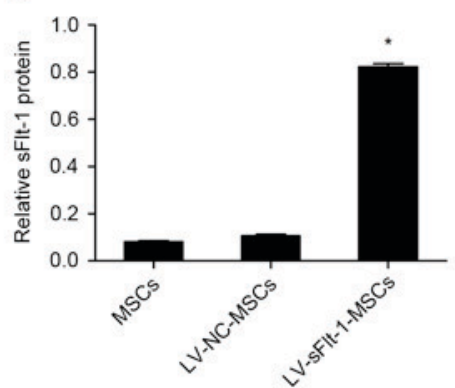

$\mathrm{F}$

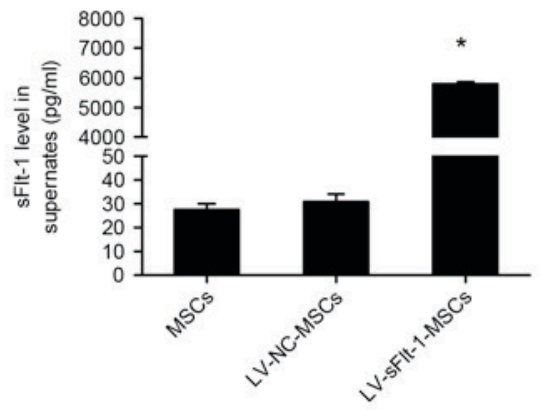

G

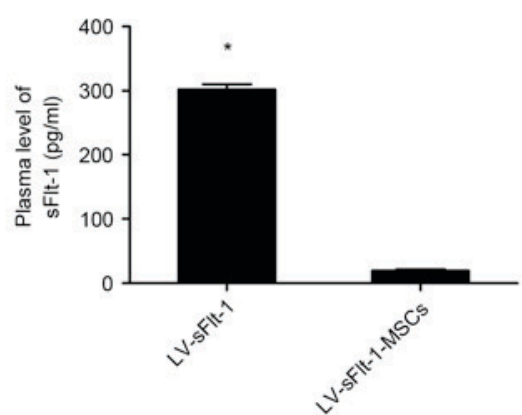

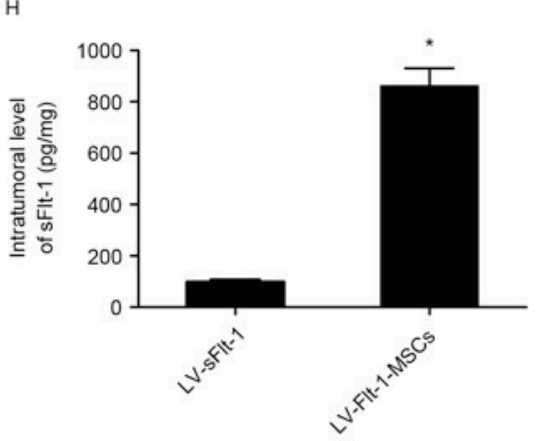

Figure 1. Construction of LV-sFlt-1-MSCs and expression of sFlt-1 in vitro and in vivo. (A) Construction of LV-sFlt-1-MSCs and LV-NC-MSCs by lentiviral transduction. Cells were observed under a fluorescence microscope at $72 \mathrm{~h}$ post-transfection; magnification, x200. (B) sFlt-1 mRNA expression was detected by polymerase chain reaction and (C) semi-quantified; LV-sFlt-1-MSCs overexpressed sFlt-1 mRNA. (D) sFlt-1 protein expression was detected by western blotting and (E) quantified by densitometry; LV-sFlt-1-MSCs significantly overexpressed sFlt-1 protein. (F) The secreted sFlt-1 levels in culture supernatants of LV-sFlt-1-MSCs, LV-NC-MSCs and untreated-MSCs were detected by ELISA. *P<0.05 vs. MSCs and LV-NC-MSCs. (G) Plasma levels of human sFlt-1 were also determined by ELISA. (H) The intratumoral levels of human sFlt-1 were determined by ELISA. "P<0.05 vs. LV-sFlt-1-MSCs. Data are presented as the mean \pm standard error of the mean. LV, lentivirus; MSCs, mesenchymal stem cells; NC, negative control; sFlt-1, soluble fms-like tyrosine kinase-1.

Tropism of MSCs for HCC in vivo. MSCs are capable of homing to the tumor microenvironment following systemic injection. To investigate the distribution of MSCs in vivo, SMMC-7721 cells stained with DiI $(10 \mu \mathrm{M})$ were subcutaneously injected into the rear of mice. A total of 2 weeks later, LV-NC-MSCs marked with GFP were intravenously injected into the tail vein tumor-bearing mice. At 7 days, the subcutaneous tumor tissues, livers, lungs, hearts, kidneys and brains were removed and cut into fresh-frozen sections, and examined under a fluorescence microscope. LV-NC-MSCs primarily migrated to the subcutaneous tumor mass, rather than other organs, including the heart, spleen and brain (Fig. 2C).

LV-sFlt-1-MSCs inhibit tumor growth and prolong survival in an HCC mouse model. To evaluate the efficacy of LV-sFlt-1-MSCsininhibiting the progress of HCC,SMMC-7721 cells were used to establish a xenograft model as aforementioned (Fig. 3A). The average tumor volume in the control group treated with PBS was $457.7 \pm 41.9 \mathrm{~mm}^{3}$ (Fig. 3B). Tumor size in the mice treated with LV-sFlt-1-MSCs $\left(86.6 \pm 6.0 \mathrm{~mm}^{3}\right)$ was significantly decreased compared with tumors in the control group $(\mathrm{P}<0.05)$. However, the tumor volume in the mice treated with LV-NC-MSCs $\left(505.4 \pm 30.4 \mathrm{~mm}^{3}\right)$ or LV-sFlt-1 $\left(383.8 \pm 12.9 \mathrm{~mm}^{3}\right)$ was not significantly different compared with the tumor volume in the control group (both $\mathrm{P}>0.05$ ). The tumor weight in the LV-sFlt-1-MSCs group was significantly decreased compared with the PBS group $(0.120 \pm 0.008$ vs. $0.326 \pm 0.010 \mathrm{~g} ; \mathrm{P}<0.05)$; whereas the tumor weight in the group treated with LV-NC-MSCs was $0.322 \pm 0.008 \mathrm{~g}(\mathrm{P}>0.05$ vs. PBS), and the weight in the control group LV-sFlt-1 was $0.286 \pm 0.012 \mathrm{~g}(\mathrm{P}>0.05 \mathrm{vs}$. PBS $)$. The results of the present study suggested that systemic injection of LV-sFlt-1-MSCs may inhibit tumor growth in an HCC mouse model.

The remaining tumor-bearing mice ( $n=5 /$ group) were monitored to examine the survival rate (Fig. 3D). The survival rate of tumor-bearing mice was monitored from the date that mice were treated with PBS, LV-NC-MSCs, LV-sFlt-1 or LV-sFlt-1-MSCs (days post-injection). The median survival of mice treated with LV-sFlt-1-MSCs was significantly increased compared with the survival in the control group treated with 

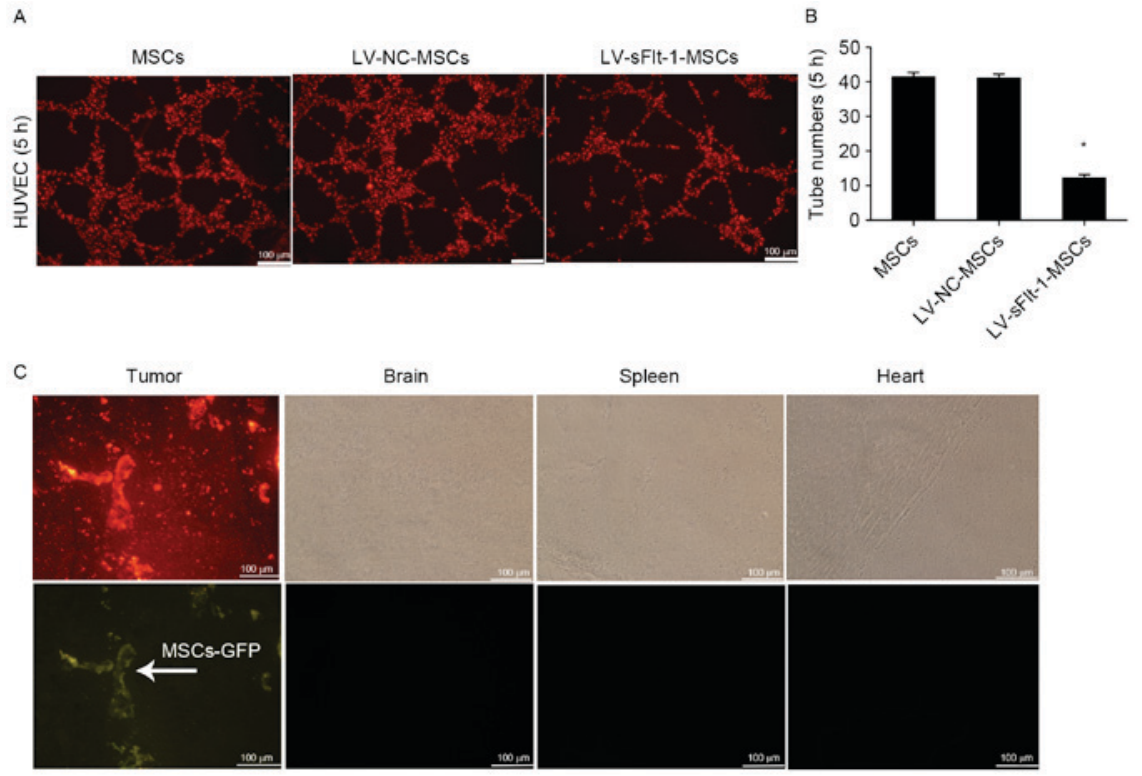

Figure 2. Effects of sFlt-1 on tube formation and distribution of MSCs in vivo. (A) HUVECs stained with DiI were co-cultured with three MSC cell lines, and tube formation was observed under a fluorescence microscope at $5 \mathrm{~h}$ (magnification, x100). (B) Tube formation was significantly decreased in the group co-cultured with LV-sFlt-1-MSCs (12.2 \pm 0.9$)$ compared with the group co-cultured with LV-NC-MSCs $(41.0 \pm 1.2)$ or MSCs $(41.4 \pm 1.3)$. "P<0.05 vs. LV-NC-MSCs. (C) SMMC-7721 stained with DiI $(10 \mu \mathrm{M})$ were used to establish a subcutaneous hepatocellular carcinoma mouse model. LV-NC-MSCs marked with GFP were intravenously injected into tumor-bearing mice. Following 7 days, LV-NC-MSCs had predominantly migrated to the subcutaneous tumor mass, rather than other organs (including the brain, spleen an heart). GFP, green fluorescent protein; HUVEC, human umbilical vein endothelial cells; LV, lentivirus; MSCs, mesenchymal stem cells; NC, negative control; sFlt-1, soluble fms-like tyrosine kinase-1.

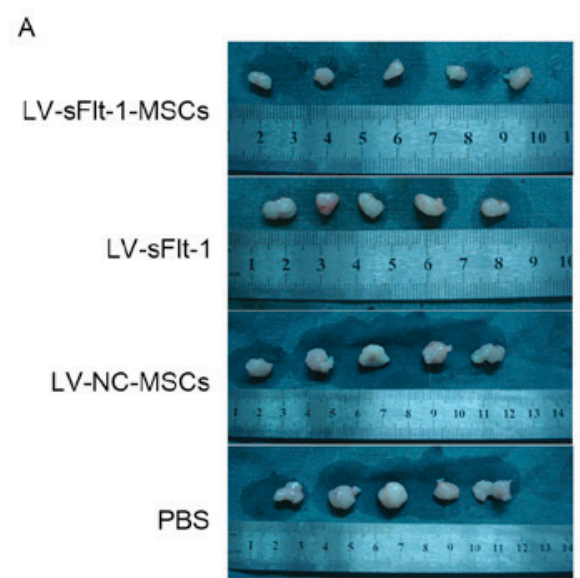

C

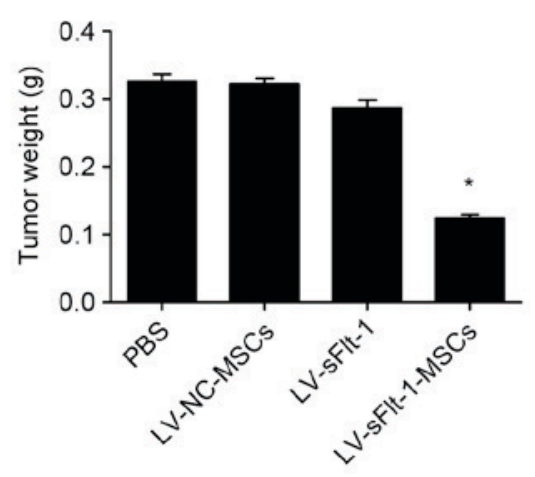

B

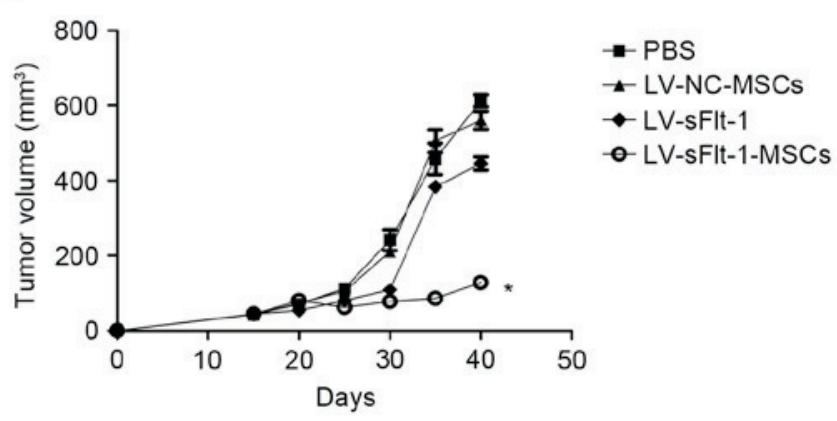

D

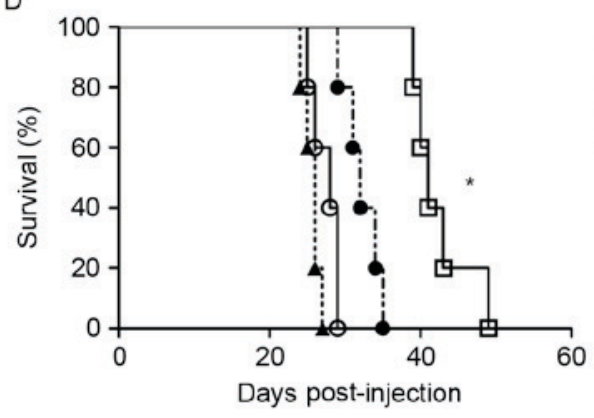

- PBS

๑ LV-NC-MSCs

- LV-sFIt-1

廿LV-sFIt-1-MSCs

Figure 3. Therapeutic effects of LV-sFlt-1-MSCs in a subcutaneous HCC model. SMMC-7721 cells were used to establish a subcutaneous HCC model. (A) When the diameter of the tumor nodule reached $0.5 \mathrm{~cm}$, tumor-bearing mice received an injection of PBS, LV-NC-MSCs, LV-sFlt-1 or LV-sFlt-1-MSCs. Following treatment for 3 weeks, the mice were sacrificed and images were captured. (B) When the diameter of the tumor nodule reached $0.5 \mathrm{~cm}$, (on day 15 post-implantation) tumor volumes were measured every 5 days. (C) Tumor tissues were removed, and tumor weights were measured. The tumor weight in the LV-sFlt-1-MSCs group was decreased compared with the PBS group. (D) Survival curves were constructed using the Kaplan-Meier method, and a log-rank test was used to evaluate significant differences. "P<0.05 vs. PBS. HCC, hepatocellular carcinoma; LV, lentivirus; MSCs, mesenchymal stem cells; NC, negative control; sFlt-1, soluble fms-like tyrosine kinase-1. 


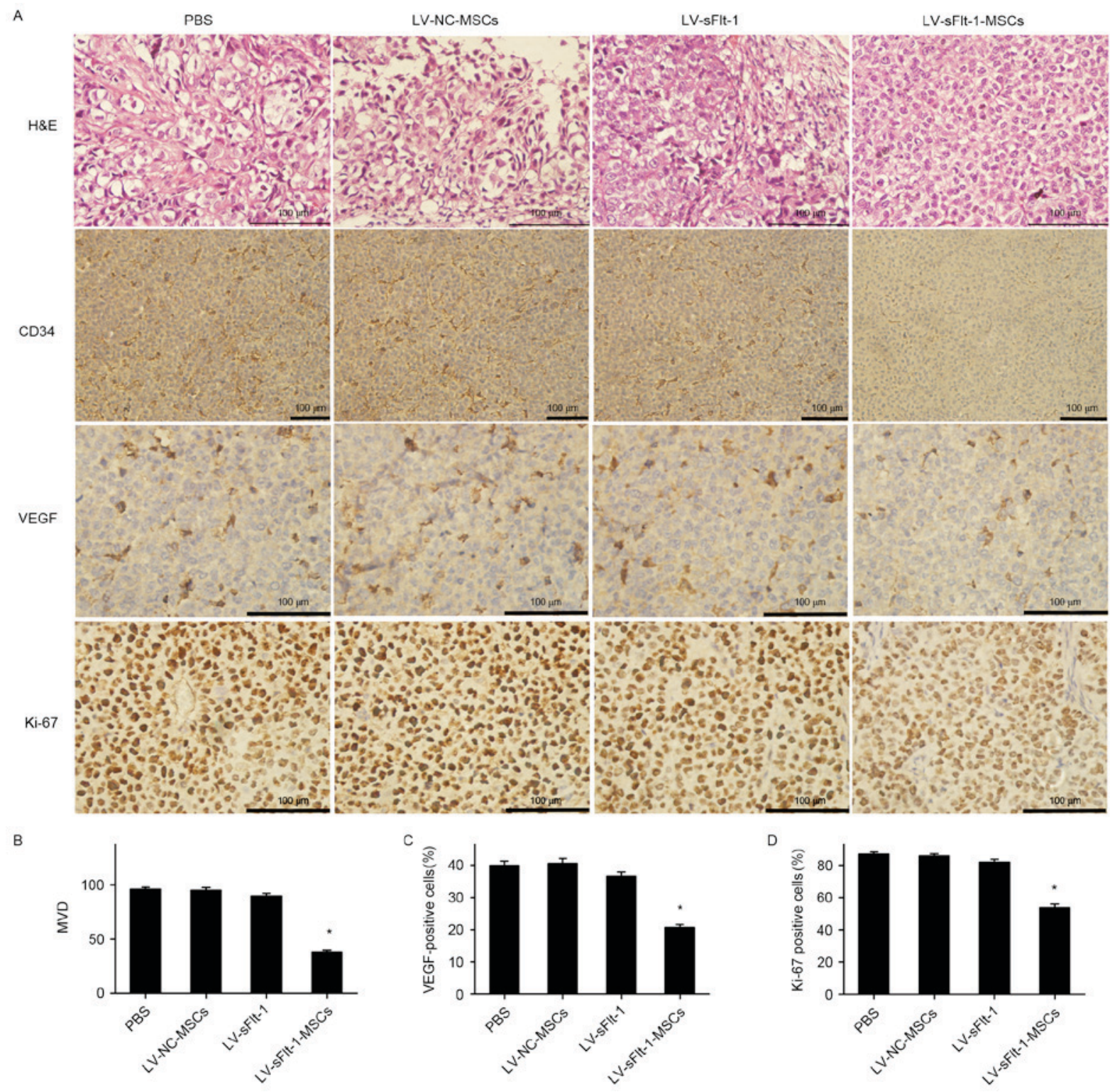

Figure 4. Immunohistochemical analysis of CD34, Ki-67 and VEGF expression. (A) Tumor sections were stained with H\&E (magnification, x400), CD34 antibody (magnification, x200), Ki-67 antibody (magnification, x400) and VEGF antibody (magnification, x400). Representative images are shown. (B) MVD was analyzed by calculating the number of CD34-positive microvessels from five randomly selected fields. (C) Protein expression of VEGF was suppressed in the mice treated with LV-sFlt-1-MSCs compared with the control group. (D) The proliferation index was determined as the number of Ki-67-positive (brown) cells/the number of total cells x 100 in five randomly selected fields. "P<0.05 vs. PBS. CD34, cluster of differentiation 34; H\&E, hematoxylin and eosin; Ki-67, proliferation marker protein Ki-67; LV, lentivirus; MSCs, mesenchymal stem cells; MVD, microvessel density; NC, negative control; sFlt-1, soluble fms-like tyrosine kinase-1; VEGF, vascular endothelial growth factor.

PBS (41 vs. 26 days; $\mathrm{P}<0.05$ ), whereas no significant differences were identified when comparing the median survival of mice treated with LV-NC-MSCs (28 days; P>0.05) or LV-sFlt-1 (32 days; P>0.05) with the survival of mice in the control group. In the present study, the results suggested that LV-sFlt-MSCs prolonged the survival time of tumor-bearing mice compared with the control group.

Anti-angiogenesis effects of LV-sFlt-1-MSCs in vivo. LV-sFlt-1-MSCs was demonstrated to suppress HCC growth through the inhibition of angiogenesis. As presented in Fig. 4, a significant decrease in MVD was observed in the group treated with LV-sFlt-1-MSCs compared with the control group treated with PBS $(38.3 \pm 1.7$ vs. $96.1 \pm 2.0 ; \mathrm{P}<0.05)$.
VEGF serves a major role in angiogenesis. Previous results suggested that sFlt-1 may inhibit tube formation and angiogenesis (10), and the present study evaluated the effects of sFlt-1 on VEGF expression by immunohistochemical analysis. The ratio of VEGF-positive staining cells to non-staining cells in the mice treated with LV-sFlt-1-MSCs was significantly decreased compared with the ratio in the control group treated with PBS (20.7 \pm 0.9 vs. $39.9 \pm 1.4 \%, \mathrm{P}<0.05$; Fig. 4C).

Effects of LV-sFlt-1-MSCs on cell proliferation in HCC. To determine whether LV-sFlt-1-MSCs were able to inhibit cell proliferation, the proliferation index, which was measured by $\mathrm{Ki}-67$ staining, was examined by immunohistochemical analysis. The average proliferation index in five random fields 
was $53.7 \pm 2.3 \%$ in the group treated with LV-sFlt-1-MSCs, $86.9 \pm 1.3 \%$ in the control group, $85.9 \pm 1.2 \%$ in the group treated with LV-NC-MSCs and $81.9 \pm 1.9 \%$ in the group treated with LV-sFlt-1 (Fig. 4D). A significant decrease in the proliferation rate was observed in the group treated with LV-sFlt-1-MSCs compared with the control group $(\mathrm{P}<0.05)$.

\section{Discussion}

The present study demonstrated that MSCs engineered to secrete sFlt-1 were able to secrete sFlt-1 efficiently and inhibit tube formation of HUVECs in vitro. Additionally, in vivo experiments indicated that, following intravenous injection, LV-sFlt-1-MSCs migrated to tumor tissues and inhibited tumor growth through anti-angiogenesis effects.

The weight of mice did not decrease following systemic injection of LV-sFlt-1-MSCs, whereas the weight of mice in the LV-sFlt-1 treatment group exhibited a transient decline. An apparent advantage of MSCs as delivery vehicles is immune privilege, which is a prerequisite for allogeneic treatment. The immune privilege of MSCs may be attributed to decreased expression of MHC class I surface markers and a lack of the co-stimulatory molecules CD40, CD80 and CD86 (25). Therefore, MSCs modified by therapeutic genes may not induce host immune response.

In the present investigation, MSCs were demonstrated to migrate to tumor tissues rather than other organs, such as the heart, spleen or brain. In addition, the intratumoral level of sFlt-1 expression in the LV-sFlt-1-MSCs group was increased compared with the LV-sFlt-1 group, whereas the plasma level of sFlt-1 in the LV-sFlt-1-MSCs group was decreased. The results of the present study further confirmed the tropism of MSCs towards tumor sites. Despite previous studies that MSCs are able to migrate to tumor sites (26-28), the molecular mechanisms underlying that migration have not been completely elucidated. Several previous studies have indicated that the tropism of MSCs may be associated with a variety of growth factors and cytokines, including hepatocyte growth factor, interleukin-6, monocyte chemotactic protein-1 and stromal-derived growth factor-1 (29-31). MSCs are thought to express chemokine receptors, including $\mathrm{C}-\mathrm{C}$ chemokine receptor type $2, \mathrm{C}-\mathrm{X}-\mathrm{C}$ chemokine receptor type 4 and hepatocyte growth factor receptor, which may be associated with the migratory property of MSCs (31-33). The possible chemotactic factors that may be involved merit further investigation.

Solid tumor growth is primarily dependent on angiogenesis. VEGF, a major angiogenic factor, has been identified to stimulate endothelial cell proliferation, migration and tube formation by interacting with VEGFR (34). VEGFR-1 is considered to be a decoy that negatively regulates the activation of VEGFR-2 by binding to intact VEGF (8). Targeting the VEGF/VEGFR signaling pathway has been used in anti-angiogenesis therapies $(35,36)$. It has been reported that sFlt-1 may suppress angiogenesis by forming inactive heterodimers with VEGFR-2 or sequestering free ligands (37). Consequently, sFlt-1 has been used as a therapeutic agent for inhibiting angiogenesis $(12,38)$. In the present study, it was demonstrated that LV-sFlt-1-MSCs suppressed tube formation of HUVECs in vitro. Spontaneous metastasis rarely happens when an HCC subcutaneous model is established (39). In previous studies, researchers monitored the survival time of subcutaneously-implanted tumor-bearing mice $(40,41)$ and revealed that subcutaneously-implanted tumor-bearing mice could also die because of the tumor burden (42). Notably, MSCs engineered to secrete sFlt-1 exhibited marked antitumor effects through the inhibition of angiogenesis, and prolonged survival in an HCC mouse model.

In conclusion, the results of the present study suggested that MSCs engineered to secrete sFlt-1 exerted potent anti-angiogenic effects in HCC. The strategy for using MSCs as delivery vehicles to treat $\mathrm{HCC}$ has numerous prospective applications. In addition to sFlt-1, additional therapeutic agents may also be delivered to treat HCC by modifying the MSCs.

\section{Acknowledgements}

The present study was supported by grants from the Natural Science Foundation of Fujian Province (grant no. 2014J01323) and The Science and Technology Project of Fujian Province (grant no. 2014Y0057).

\section{References}

1. Fernández M, Semela D, Bruix J, Colle I, Pinzani M and Bosch J: Angiogenesis in liver disease. J Hepatol 50: 604-620, 2009.

2. Carmeliet P and Jain RK: Molecular mechanisms and clinical applications of angiogenesis. Nature 473: 298-307, 2011.

3. Miura H, Miyazaki T, Kuroda M, Oka T, Machinami R, Kodama T, Shibuya M, Makuuchi M, Yazaki Y and Ohnishi S: Increased expression of vascular endothelial growth factor in human hepatocellular carcinoma. J Hepatol 27: 854-861, 1997.

4. Yamaguchi R, Yano H, Iemura A, Ogasawara S, Haramaki M and Kojiro M: Expression of vascular endothelial growth factor in human hepatocellular carcinoma. Hepatology 28: 68-77, 1998.

5. Terman BI, Carrion ME, Kovacs E, Rasmussen BA, Eddy RL and Shows TB: Identification of a new endothelial cell growth factor receptor tyrosine kinase. Oncogene 6: 1677-1683, 1991.

6. Pajusola K, Aprelikova O, Korhonen J, Kaipainen A, Pertovaara L, Alitalo R and Alitalo K: FLT4 receptor tyrosine kinase contains seven immunoglobulin-like loops and is expressed in multiple human tissues and cell lines. Cancer Res 52: 5738-5743, 1992.

7. Shibuya M: Structure and dual function of vascular endothelial growth factor receptor-1 (Flt-1). Int J Biochem Cell Biol 33: 409-420, 2001

8. Shibuya M and Claesson-Welsh L: Signal transduction by VEGF receptors in regulation of angiogenesis and lymphangiogenesis. Exp Cell Res 312: 549-560, 2006.

9. Kendall RL, Wang G and Thomas KA: Identification of a natural soluble form of the vascular endothelial growth factor receptor, FLT-1, and its heterodimerization with KDR. Biochem Biophys Res Commun 226: 324-328, 1996.

10. Koga J, Matoba T, Egashira K, Kubo M, Miyagawa M, Iwata E, Sueishi K, Shibuya M and Sunagawa K: Soluble Flt-1 gene transfer ameliorates neointima formation after wire injury in flt-1 tyrosine kinase-deficient mice. Arterioscler Thromb Vasc Biol 29: 458-464, 2009.

11. Bagley RG, Kurtzberg L, Weber W, Nguyen TH, Roth S, Krumbholz R, Yao M, Richards B, Zhang M, Pechan P, et al: sFLT01: A novel fusion protein with antiangiogenic activity. Mol Cancer Ther 10: 404-415, 2011.

12. Krishnan B, Torti FM, Gallagher PE and Tallant EA: Angiotensin-(1-7) reduces proliferation and angiogenesis of human prostate cancer xenografts with a decrease in angiogenic factors and an increase in sFlt-1. Prostate 73: 60-70, 2013.

13. Owen LA, Uehara H, Cahoon J, Huang W, Simonis J and Ambati BK: Morpholino-mediated increase in soluble Flt-1 expression results in decreased ocular and tumor neovascularization. PLoS One 7: e33576, 2012.

14. Tipnis S, Viswanathan C and Majumdar AS: Immunosuppressive properties of human umbilical cord-derived mesenchymal stem cells: Role of B7-H1 and IDO. Immunol Cell Biol 88: 795-806, 2010 . 
15. Kidd S, Spaeth E, Dembinski JL, Dietrich M, Watson K, Klopp A, Battula VL, Weil M, Andreeff M and Marini FC: Direct evidence of mesenchymal stem cell tropism for tumor and wounding microenvironments using in vivo bioluminescent imaging. Stem Cells 27: 2614-2623, 2009.

16. Sasportas LS, Kasmieh R, Wakimoto H, Hingtgen S, van de Water JA, Mohapatra G, Figueiredo JL, Martuza RL, Weissleder R and Shah K: Assessment of therapeutic efficacy and fate of engineered human mesenchymal stem cells for cancer therapy. Proc Natl Acad Sci USA 106: 4822-4827, 2009.

17. Loebinger MR, Kyrtatos PG, Turmaine M, Price AN, Pankhurst Q, Lythgoe MF and Janes SM: Magnetic resonance imaging of mesenchymal stem cells homing to pulmonary metastases using biocompatible magnetic nanoparticles. Cancer Res 69: 8862-8867, 2009.

18. Ren C, Kumar S, Chanda D, Chen J, Mountz JD and Ponnazhagan S: Therapeutic potential of mesenchymal stem cells producing IFN-alpha in a mouse melanoma lung metastasis model. Stem Cells 26: 2332-2338, 2008

19. Nakamura K, Ito Y, Kawano Y, Kurozumi K, Kobune M, Tsuda H, Bizen A, Honmou O, Niitsu Y and Hamada H: Antitumor effect of genetically engineered mesenchymal stem cells in a rat glioma model. Gene Ther 11: 1155-1164, 2004.

20. Gao Y, Yao A, Zhang W, Lu S, Yu Y, Deng L, Yin A, Xia Y, Sun B and Wang $\mathrm{X}$ : Human mesenchymal stem cells overexpressing pigment epithelium-derived factor inhibit hepatocellular carcinoma in nude mice. Oncogene 29: 2784-2794, 2010.

21. Li X, Lu Y, Huang W, Xu H, Chen X, Geng Q, Fan H, Tan Y, Xue $G$ and Jiang $X$ : In vitro effect of adenovirus-mediated human gamma interferon gene transfer into human mesenchymal stem cells for chronic myelogenous leukemia. Hematol Oncol 24: 151-158, 2006.

22. Chen Q, Cheng P, Yin T, He H, Yang L, Wei Y and Chen X: Therapeutic potential of bone marrow-derived mesenchymal stem cells producing pigment epithelium-derived factor in lung carcinoma. Int J Mol Med 30: 527-534, 2012.

23. Zeng Y, Opeskin K, Goad J and Williams ED: Tumor-induced activation of lymphatic endothelial cells via vascular endothelial growth factor receptor-2 is critical for prostate cancer lymphatic metastasis. Cancer Res 66: 9566-9575, 2006.

24. Weidner N, Semple JP, Welch WR and Folkman J: Tumor angiogenesis and metastasis - correlation in invasive breast carcinoma N Engl J Med 324: 1-8, 1991.

25. Kidd S, Spaeth E, Klopp A, Andreeff M, Hall B and Marini FC: The (in) auspicious role of mesenchymal stromal cells in cancer: Be it friend or foe. Cytotherapy 10: 657-667, 2008

26. Kim SM, Oh JH, Park SA, Ryu CH, Lim JY, Kim DS, Chang JW, $\mathrm{Oh} \mathrm{W}$ and Jeun SS: Irradiation enhances the tumor tropism and therapeutic potential of tumor necrosis factor-related apoptosis-inducing ligand-secreting human umbilical cord blood-derived mesenchymal stem cells in glioma therapy. Stem Cells 28: 2217-2228, 2010.

27. Zou W, Zheng H, He TC, Chang J, Fu YX and Fan W: LIGHT delivery to tumors by mesenchymal stem cells mobilizes an effective antitumor immune response. Cancer Res 72: 2980-2989, 2012.

28. Ho IA, Toh HC, Ng WH, Teo YL, Guo CM, Hui KM and Lam PY: Human bone marrow-derived mesenchymal stem cells suppress human glioma growth through inhibition of angiogenesis. Stem Cells 31: 146-155, 2013.
29. Wels J, Kaplan RN, Rafii S and Lyden D: Migratory neighbors and distant invaders: Tumor-associated niche cells. Genes Dev 22: 559-574, 2008.

30. Studeny M, Marini FC, Dembinski JL, Zompetta C, Cabreira-Hansen M, Bekele BN, Champlin RE and Andreeff M: Mesenchymal stem cells: Potential precursors for tumor stroma and targeted-delivery vehicles for anticancer agents. J Natl Cancer Inst 96: 1593-1603, 2004.

31. Son BR, Marquez-Curtis LA, Kucia M, Wysoczynski M, Turner AR, Ratajczak J, Ratajczak MZ and JanowskaWieczorek A: Migration of bone marrow and cord blood mesenchymal stem cells in vitro is regulated by stromal-derived factor-1-CXCR4 and hepatocyte growth factor-c-met axes and involves matrix metalloproteinases. Stem Cells 24: 1254-1264, 2006.

32. Song $\mathrm{C}$ and Li G: CXCR4 and matrix metalloproteinase- 2 are involved in mesenchymal stromal cell homing and engraftment to tumors. Cytotherapy 13: 549-561, 2011.

33. Klopp AH, Spaeth EL, Dembinski JL, Woodward WA, Munshi A, Meyn RE, Cox JD, Andreeff M and Marini FC: Tumor irradiation increases the recruitment of circulating mesenchymal stem cells into the tumor microenvironment. Cancer Res 67: 11687-11695, 2007.

34. Shibuya M: Vascular endothelial growth factor and its receptor system: Physiological functions in angiogenesis and pathological roles in various diseases. J Biochem 153: 13-19, 2013.

35. Zeng Z, Huang WD, Gao Q, Su ML, Yang YF, Liu ZC and Zhu BH: Arnebin-1 promotes angiogenesis by inducing eNOS, VEGF and HIF- $1 \alpha$ expression through the PI3K-dependent pathway. Int J Mol Med 36: 685-697, 2015.

36. Kim SL, Lee ST, Trang KT, Kim SH, Kim IH, Lee SO, Kim DG and Kim SW: Parthenolide exerts inhibitory effects on angiogenesis through the downregulation of VEGF/VEGFRs in colorectal cancer. Int J Mol Med 33: 1261-1267, 2014.

37. Takei Y, Mizukami H, Saga Y, Yoshimura I, Hasumi Y, Takayama T, Kohno T, Matsushita T, Okada T, Kume A, et al: Suppression of ovarian cancer by muscle-mediated expression of soluble VEGFR-1/Flt-1 using adeno-associated virus serotype 1-derived vector. Int J Cancer 120: 278-284, 2007.

38. Justiniano SE, Elavazhagan $S$, Fatehchand $K$, Shah $P$, Mehta $P$ Roda JM, Mo X, Cheney C, Hertlein E, Eubank TD, et al: Fcy receptor-induced soluble vascular endothelial growth factor receptor-1 (VEGFR-1) production inhibits angiogenesis and enhances efficacy of antitumor antibodies. J Biol Chem 288: 26800-26809, 2013.

39. Killion JJ, Radinsky R and Fidler IJ: Orthotopic models are necessary to predict therapy of transplantable tumors in mice. Cancer Metastasis Rev 17: 279-284, 1999.

40. Wang J, Xu L, Zeng W, Hu P, Zeng M, Rabkin SD and Liu R: Treatment of human hepatocellular carcinoma by the oncolytic herpes simplex virus G47delta. Cancer Cell Int 14: 83, 2014.

41. Schmitz V, Raskopf E, Gonzalez-Carmona MA, Vogt A, Rabe C, Leifeld L, Kornek M, Sauerbruch T and Caselmann W: Plasminogen fragment K1-5 improves survival in a murine hepatocellular carcinoma model. Gut 56: 271-278, 2007.

42. Zhu LM, Shi DM, Dai Q, Cheng XJ, Yao WY, Sun PH, Ding Y, Qiao MM, Wu YL, Jiang SH and Tu SP: Tumor suppressor XAF1 induces apoptosis, inhibits angiogenesis and inhibits tumor growth in hepatocellular carcinoma. Oncotarget 5 5403-5415, 2014 